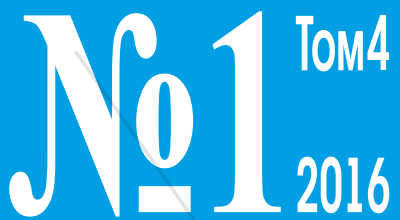

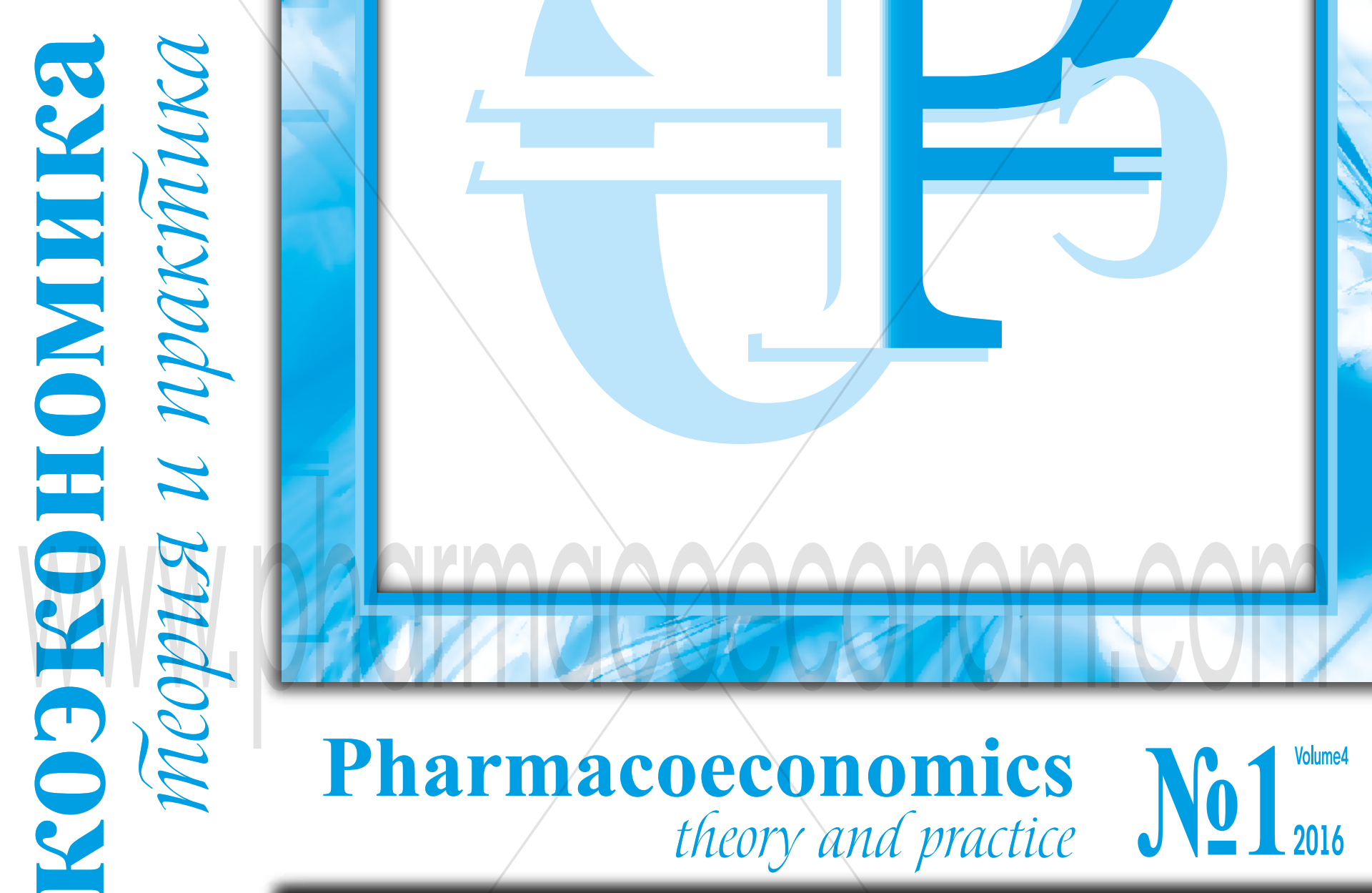

$\square$ МЕТОДОЛОГИЧЕСКИЕ ОСНОВЫ ФАРМАКОЭКОНОМИЧЕСКОГО МОДЕЛИРОВАНИЯ

$\square$ РЕЗУЛЬТАТЫ РОССИЙСКИХ ФАРМАКОЭКОНОМИЧЕСКИХ ИССЛЕДОВАНИЙ

口 МАТЕРИАЛЫ Х НАЦИОНАЛЬНОГО КОНГРЕССА С МЕЖДУНАРОДНЫМ УЧАСТИЕМ «РАЗВИТИЕ ФАРМАКОЭКОНОМИКИ И ФАРМАКОЭПИДЕМИОЛОГИИ В РОССИЙСКОЙ ФЕДЕРАЦИИ» 4-5 апреля 2016 г., г. Нижний Новгород 


\title{
ОЦЕНКА ЛЕКАРСТВЕННОЙ ТЕРАПИИ ПАЦИЕНТОВ С ТУБЕРОЗНЫМ СКЛЕРОЗОМ С ПОМОЩЬЮ МЕТОДОЛОГИИ МУЛЬТИКРИТЕРИАЛЬНОГО АНАЛИЗА ПРИНЯТИЯ РЕШЕНИЙ
}

\author{
Ягудина Р.И. ${ }^{1}$, Серпик В.Г. ${ }^{1}$, Дорофеева М.Ю. ${ }^{2}$ \\ ${ }^{1}$ ГБОУ ВПО «Первый МГМУ им. И.М. Сеченова» Минздрава РФ, Москва \\ ${ }^{2}$ «Научно-исследовательский клинический институт педиатрии имени академика \\ Ю.Е. Вельтищева» ГБОУ ВПО РНИМУ им. Н.И. Пирогова
}

\section{Резюме:}

Туберозный склероз - редкое жизнеугрожающее заболевание, сопровождающееся тяжелым поражением функциональных систем организма, эффективность существовавшего лечения которого до недавнего времени была весьма ограничена. Появление инновационного препарата эверолимус, воздействующего на патогенез заболевания, создает новые возможности в лечении пациентов с туберозным склерозом. Однако, его значительная стоимость и орфанный статус требуют особого отношения при включении в перечни лекарственных препаратов в рамках принятой в настоящее время процедуры, включающей классическую фрармакоэкономическую оценку. $B$ настоящее время для оценки орфанных лекарственных препаратов активно обсуждается новый подход, базирующийся на мультикритериальном анализе принятия решений. Мультикритериальный анализ принятия решений, учитывая фармакоэкономическую оценку, позволяетпринимать вовнимание и другие важные критерии, характеризующие проблему орфанных лекарственных препаратов. В результате, лицам, принимающим решения, предоставляется информация, всесторонне описывающая рассматриваемую проблематику, что создает предпосылки для взвешенных решений при включении в соответствующие перечни лекарственных средств. В процессе данного мультикритериального анализа принятия решений был определен список критериев, по которым оценивалось заболевание туберозный склероз и лекарственный препарат эверолимус. На основе списка был разработан опросник и проведено интервьюирование респондентов для оценки заболевания и препарата. При этом, оценка проводилась последовательно: в первой части опросника определялась значимость самих критериев для респондентов; вторая часть опросника оценивала уже само заболевание и лекарственный препарат по определенным критериям. На заключительном этапе рассчитывалась интегральная оценка по каждому из критериев.

В рамках проведенного исследования были выделены две группы критериев, характеризующих объекты исследования - нозологию и препарат (по пять критериев в каждой группе). К группе критериев, характеризующих заболевание, были отнесены: клиническая тяжесть; экономическое бремя; критерий, отражающий денежные средства, инвестированные в развитие инфраструктуры лечения заболевания (создание регистра пациентов, обучение клинических специалистов, школы пациентов); социальный и эпидемиологический критерии. Среди критериев, характеризующих препарат, были выделены следующие клиническая эфффективность (безопасность); фрармакоэкономическая оценка; инновационность препарата; критерии, отражающие этические аспекты использования препарата и характеризующие удобство его применения.

Диапазон возможных значений интегральной оценки варьировался от 1 балла до 35 баллов. Максимальная интегральная оценка по критериям, характеризующим заболевание, была присвоена клиническому критерию. B группе критериев, характеризующих лекарственный препарат, максимальное значение было получено также для критерия клинической эфффективности препарата: по мнению пациентов оно составило 29,1 балла; по мнению клинических специалистов - 32,4 балла; специалистов по оценке технологий здравоохранения - 30,4 балла; организаторов здравоохранения - 28,2 балла. Необходимо отметить также высокую интегральную оценку организаторами здравоохранения социального критерия для туберозного склероза. Все группы респондентов высоко оценили инновационный потенциал эверолимуса.

Полученные в ходе мультикритериального анализа принятия решений интегральные оценки свидетельствуют о наличии понимания социальной значимости рассматриваемого заболевания и востребованности пациентов с туберозным склерозом в препарате эверолимус. Результаты проведенного анализа могут быть использованы для повышения доступности препарата эверолимус для пациентов с туберозным склерозом

Ключевые слова: мультикритериальный анализ принятия решений, оценка технологий здравоохранения, редкое заболевание, орфанный препарат, туберозный склероз, эверолимус.

Туберозный склероз - это генетически детерминированное заболевание из группы факоматозов, отличающееся широким спектром клинических проявлений и прогрессирующим течением, которое приводит к сокращению продолжительности жизни и инвалидизации пациентов. Туберозный склероз относится к группе редких заболеваний с распространенностью 1:10000 [19].

Туберозный склероз характеризуется развитием множественных доброкачественных опухолей (гамартом) в различных органах, включая головной мозг, глаза, кожу, сердце, почки, печень, легкие, желудочно-кишечный тракт, эндокринную и костную системы. Постепенно прогрессируя и увеличиваясь в размерах, они нарушают функции этих органов, в ряде случаев приводя к гибели пациентов [18]

Наиболее драматические последствия заболевания, связанные с поражением центральной нервной системы, могут встречаться уже в детском возрасте. Степень выраженности поражения головного мозга (туберов и субэпидемальных узлов, которые выявляются у 95-98\% пациентов) зависит от развития эпилепсии, часто фармакорезистентной, приводящей к возникновению нарушений когнитивных функций (нарушения обучения и поведения). Развитие субэпендимальных гигантоклеточных астроцитом (СЭГА) у 20\% пациентов с туберозным склерозом может приводить к летальному исходу заболевания [18].

До недавнего времени терапия туберозного склероза носила симптоматический характер. Единственным методом терапии пациентов с жизнеугрожающими СЭГА было оперативное лечение, 
применение которого в ряде случаев противопоказано, что, фрактически, означало отсутствие лечения для данной подгруппы пациентов. В 2012 году в Российской Федерации был зарегистрирован первый лекарственный препарат (ЛП) для терапии туберозного склероза, эверолимус (Афинитор), воздействующий на патогенез заболевания и препятствующий развитию опухолей и, следовательно, предотвращающий повреждение органов [18].

Лекарственный препарат эверолимус открыл новые возможности в лечении туберозного склероза, и является жизнесохраняющей медицинской технологией, предотвращающей фатальные последствия развития СЭГА

Лечение туберозного склерозаэверолимусом носит патогенетический характер, что в соответствии с законодательством Российской Федерации позволяет отнести его к орфанным лекарственным препаратам. [1-4,17, 19]. Орфанные лекарственные препараты в силу своих особенностей высокой стоимости и узкой группы пациентов - фактически, находятся за рамками (рис. 1) существующего механизма включения препаратов в перечни лекарственных препаратов, предъявляющего жесткие требования к их фармакоэкономическому профилю.

Наиболее высокую социальную значимость приобретает проблема обеспечения пациентов с редкими заболеваниями жизнеспасающими лекарственными препаратами, в том числе и пациентов с туберозным склерозом, что требует создания новых механизмов для оценки объективности принятия решений о финансировании лекарственного обеспечения пациентов с редкими заболеваниями.

Для решения данной проблемы ведущими системами здравоохранения в мире предпринимаются попытки внедрить новый инструмент принятия решений, который учитывал бы медико-эпидемиологические особенности орфанных заболеваний мультикритериальный анализ принятия решений (MAПP, MCDA - multicriteria decision analysis).

МАПР - инструмент, используемый в целях предоставления лицам, принимающим решения, наиболее полной и релевантной информации по обсуждаемым проблемам в условиях множественной неопределенности, для принятия корректного решения.

С учетом проводимой нами научной работы по адаптации методов МАПР и их имплементации в отечественную систему здравоохранения, особый интерес вызвала возможность апробировать методику данного вида анализа для оценки применения препарата эверолимус в терапии туберозного склероза. При проведении МАПР выявлялись и учитывались дополнительные важные аспекты лекарственного обеспечения больных туберозным склерозом лекарственным препаратом эверолимус. Благодаря этому лица, принимающие решения, могут получить комплексную инсормацию о проблеме и на её основе принимать более взвешенные решения по лекарственному обеспечению пациентов.

\section{Методология мультикритериального анализа принятия решений}

Концепция МАПР в рамках оценки технологии здравоохранения (0Т3), может быть представлена следующим образом. При принятии решений в областях, характеризующихся высокими рисками и степенью неопределенности (к каковым относится и лекарственная помощь пациентам с редкими заболеваниями), необходимым становится повышение прозрачности и обоснованности процедуры принятия решений. Необходимым условием при этом является учет и формализованное отражение позиции всех заинтересованных в рассматриваемой области сторон (рис. 2) [5-16].

В настоящий момент разработано множество методологических решений для проведения МАПР. Однако, принимая во внимание, что в условиях здравоохранения Российской Федерации практическое внедрение методологии МАПР было проведено впервые, а также международный опыт, рекомендующий на этапе внедрения МАПР использование по возможности интуитивно-понятных методов, проведенное нами исследование МАПР имело следующий алгоритм.

І этапом исследования являлось выделение критериев, релевантных для рассматриваемой проблемы. Указанный этап проводился специалистами ОТЗ и фрармакоэкономики.

Критерий - это признак, имеющий количественное или качественное выражение, по которому проводится оценка исследуемого объекта.

॥ этап включал разработку опросника, с помощью которого определяли мнение респондентов о значимости выделенных ранее критериев (в целом, независимо от рассматриваемой нозологии или лекарственного препарата). Также был разработан опросник для непосредственной оценки изучаемого заболевания и лекарственного препарата по выделенным критериям.

III этап исследования решал задачу выбора групп заинтересованных сторон процесса лекарственного обеспечения пациентов с рассматриваемым заболеванием и, собственно, формирования списка

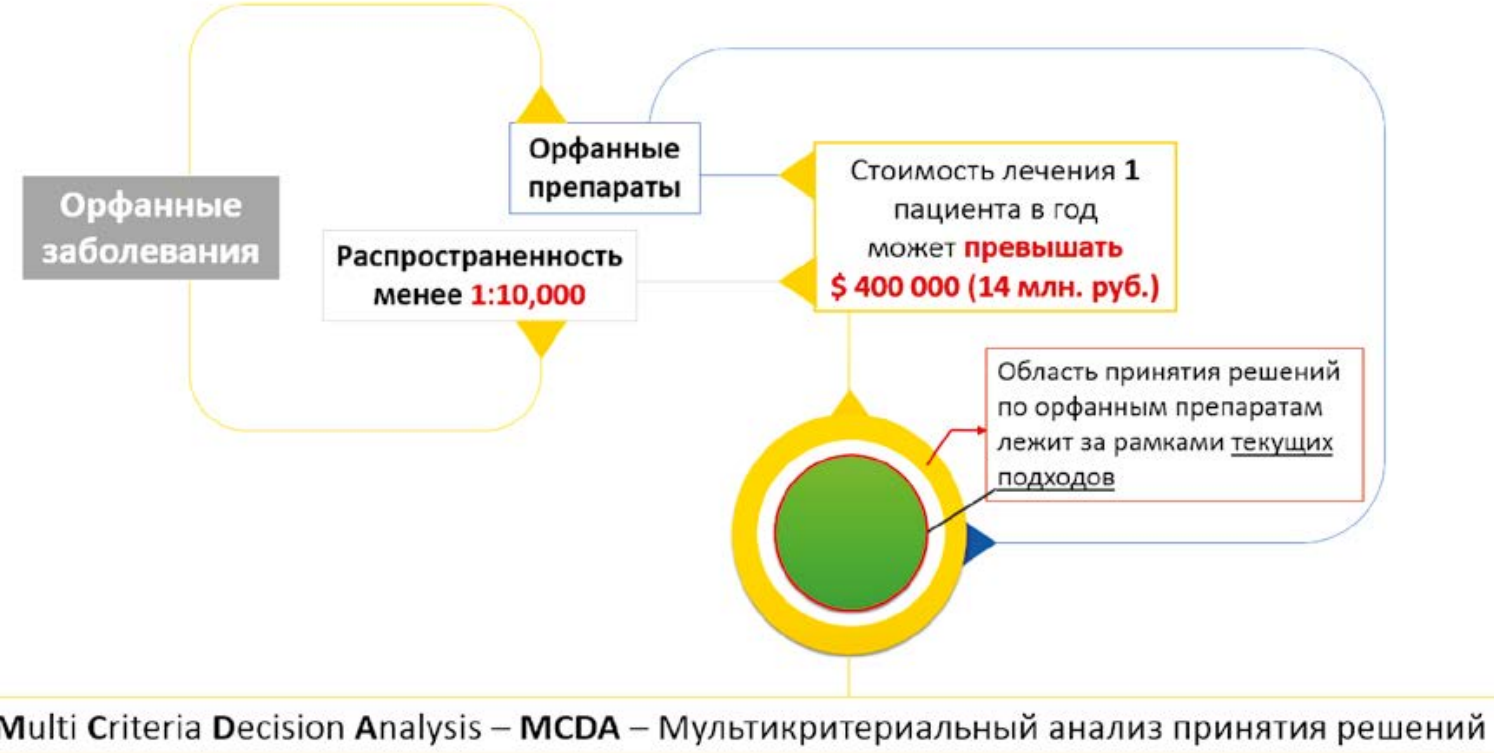

Рисунок 1. Ограниченность текущих подходов к принятию решений в области лекарственного обеспечения орфанными препаратами 


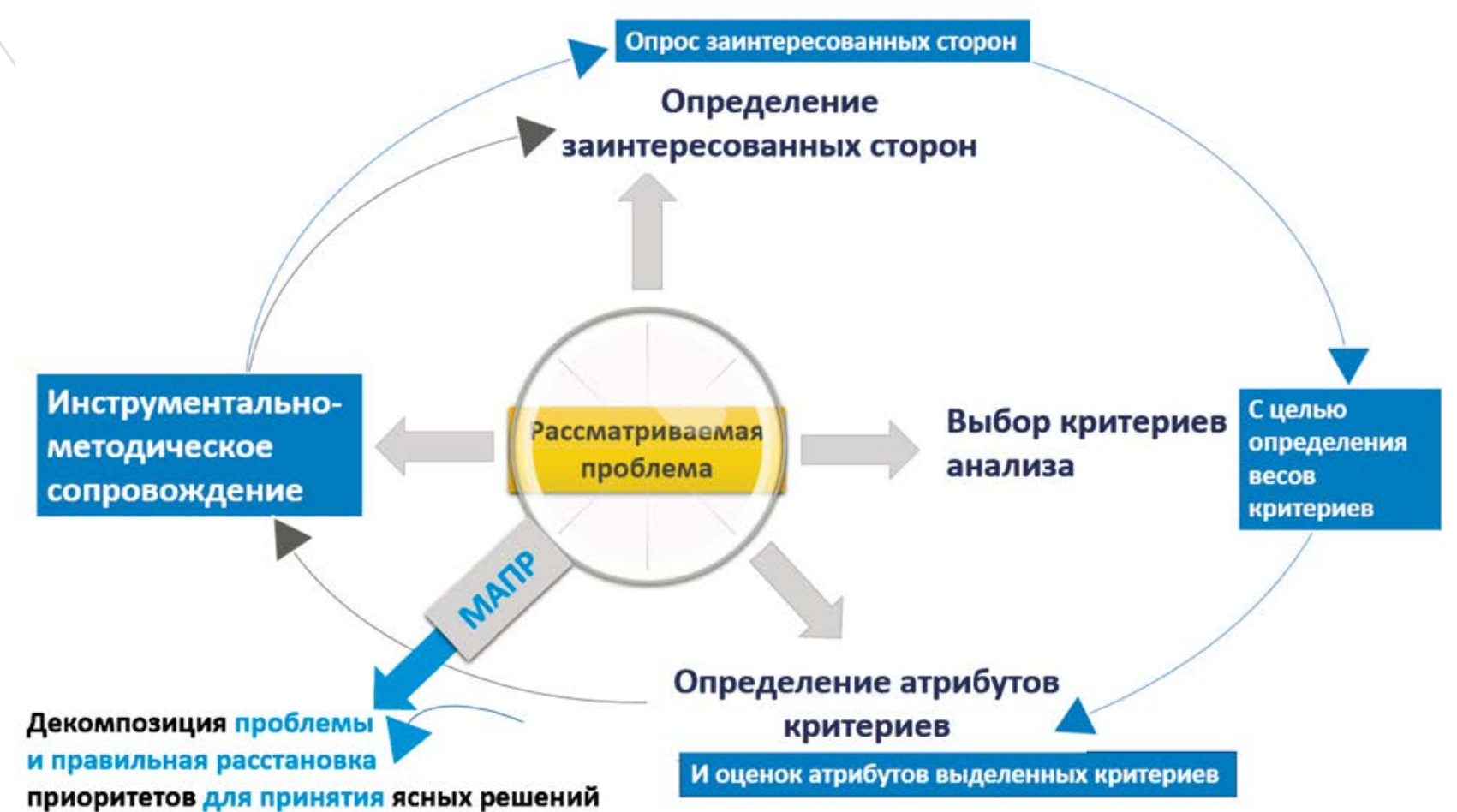

Рисунок 2. Принципиальная схема МАПР

респондентов. Основываясь на действующей в России структуре здравоохранения рассматривались следующие группы респондентов: пациенты и их представители (пациентские организации), клинические специалисты (врачи), специалисты по ОТЗ и фрармакоэкономике и организаторы здравоохранения (ЛПР).

IV этап МАПР включал заполнение респондентами разработанных опросников.

$V$ заключительный этап заключался в анализе ответов респондентов. В ходе данного этапа изучался вопрос о соотношении важности для респондентов группы критериев для заболевания и группы критериев для лекарственного препарата.

Обязательным начальным шагом $V$ этапа был расчет средних весов каждого из критериев для отдельных группы респондентов.

Вес критерия - это количественная характеристика самого критерия, которая отражает значимость конкретного критерия в сравнении с другими критериями

Формула для расчета среднего веса критерия представлена ниже:

$$
\begin{aligned}
& \bar{W}_{i}=\left(\sum_{1}^{n} w\right) / n \text { Формула (1) } \\
& \text { где: }
\end{aligned}
$$

$\bar{W}_{i}$-среднее значение веса критерия $i$;

$W$ - значение веса критерия по мнению респондента;

$n$ - число респондентов, чел.

Оценка нозологии/лекарственного препарата по критерию - это количественное или качественное выражение характеристики объекта исследования по отношению к нозологии или лекарственному препарату по заданному критерию.

Аналогичным образом по формуле 2 рассчитывались средние значения оценки респондентами заболевания туберозный склероз и лекарственного препарата эверолимус по выбранным критериям:

$\bar{a}_{i}=\left(\Sigma_{1}^{n} a\right) / n$ Формула (2) где:

$\bar{a}_{i}$-среднее значение оценки по критерию $i$;

$a$ - значение оценки по критерию і по мнению респондента;

$n$ - число респондентов, чел.

На основе рассчитанных средних значений весов критерия и оценок туберозного склероза и эверолимуса по формуле 3 определялась интегральная оценка результатов МАПР:

$\mathrm{Ia}_{\mathrm{i}}=\bar{w}_{i} \times \bar{a}_{i}$ Формула (3)

где:

$\mathrm{la}_{\mathrm{i}}$ - интегральная оценка МАПР заболевания туберозный склероз или лекарственного препарата эверолимус по критерию i;

$\bar{W}_{i}$ - среднее значение веса критерия $i$;

$\bar{a}_{i}$ - среднее значение оценки по критерию $i$.

Полученные интегральные оценки для туберозного склероза и эверолимуса по мнению различных групп респондентов подвергались сравнительному анализу с использованием двух подходов:

- Компенсирующего;

- Некомпенсирующего

При компенсирующем подходе интегральные оценки МАПР, полученные от каждой группы респондентов, суммировались:

Sla $=\sum_{1}^{i} I a_{i}$ Формула (4)

где:

Sla - сумма интегральных оценок МАПР одной группы респондентов по всем критериям;

$\mathrm{I}_{\mathrm{i}}$ - интегральная оценка МАПР заболевания туберозный склероз или лекарственного препарата эверолимус по критерию i.

После расчета суммарных значений интегральных оценок результатов МАПР для каждой из групп респондентов их сравнивали между собой. В целях оптимизации процесса МАПР в рамках проводимого исследования была разработана интерактивная модель МАПР, автоматически рассчитывающая оценки МАПР (рис. 3-5). 


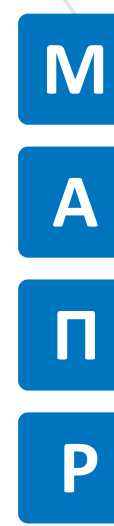

ультикритериальный

нализ

ринятия

ешений
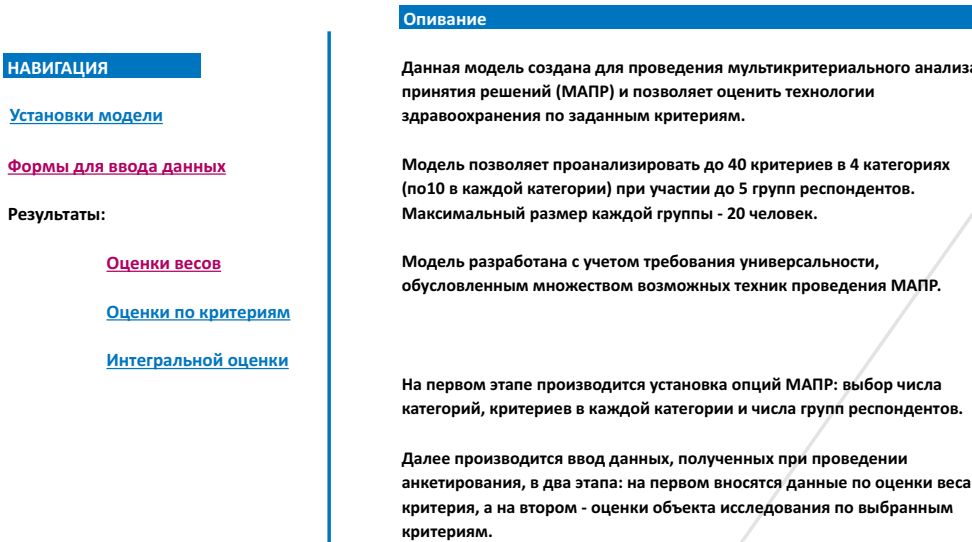

Данная модель создана для проведения мультикритериального анализа принятия решений (МАПР) и позволяет оценить технологии здравоохранения по заданным критериям.

Модель позволяет проанализировать до 40 критериев в 4 категориях (по10 в каждой категории) при участии до 5 групп респондентов. Максимальный размер каждой группы - 20 человек.

Модель разработана с учетом требования универсальности, обусловленным множеством возможных техник проведения МАПР.

На первом этапе производится установка опций МАПР: выбор числа категорий, критериев в каждой категории и числа групп респондентов. Далее производится ввод данных, полученных при проведении анкетирования, в два этапа: на первом вносятся данные по оценки веса критерия, а на втором - оценки объекта исследования по выбранным критериям.

Рисунок 3. Интерактивная модель МАПР - титульный лист

Использование некомпенсирующего подхода включало сравнение полученных значений интегральных оценок по каждому из критериев между группами респондентов. Учитывая инновационный характер исследования, ограниченное число респондентов и единичную численность объектов исследования - одного заболевания и одного лекарственного препарата, в проведении статистического анализа полученных данных не было необходимости.

Таким образом, целью проведения данной работы было проведение первого в Российской Федерации исследования по оценке терапии пациентов с туберозным склерозом лекарственным препаратом эверолимус с помощью методологии МАПР

\section{Материалы и методы}

В соответствии с изложенной методологией на первом этапе исследования нами был сформирован пул критериев МАПР, включавший в себя, как критерии для описания редкого заболевания, так и для описания орфанного лекарственного препарата.

\begin{tabular}{|c|c|}
\hline Критерий & Описание \\
\hline Клинический: & $\begin{array}{c}\text { Влияние заболевания на состояние здоровье } \\
\text { пациента (физическое, ментальное, } \\
\text { эмоциональное) }\end{array}$ \\
\hline Экономический: & $\begin{array}{c}\text { Экономические последствия заболевания } \\
\text { (включая затраты системы здравоохранения } \\
\text { на лечение заболевания, экономические } \\
\text { потери, которое несет общество в целом по } \\
\text { причине преждевременной смертности или } \\
\text { инвалидизации пациентов) }\end{array}$ \\
\hline Инвестиционный: & $\begin{array}{c}\text { Объем ресурсов, инвестированный } \\
\text { в изучение заболевания/ создание } \\
\text { инфраструктуры для достижения наилучшего } \\
\text { результата от предполагаемого лечения } \\
\text { (создание локальных и всероссийского } \\
\text { регистров пациентов, проведение школ } \\
\text { пациентов, издание информационных } \\
\text { материалов для врачей различных } \\
\text { специальностей и пациентов, и т.п.) }\end{array}$ \\
\hline Социальный: & $\begin{array}{l}\text { Насколько общество в целом считает } \\
\text { необходимым оказывать медицинскую } \\
\text { помощь пациентам с данной нозологией }\end{array}$ \\
\hline Эпидемиологический: & $\begin{array}{c}\text { Насколько распространена рассматриваемая } \\
\text { нозология в Российской Федерации }\end{array}$ \\
\hline
\end{tabular}

На следующем этапе проводимого исследования были разработань оценочные материалы для респондентов. Они включали два опросника:

- Опросник для определения весов критериев;

- Опросник для оценки туберозного склероза и его лечения по выбранным критериям.

\begin{abstract}
Критерий
Описание

Клинический:

Фармакоэкономический: $\quad$ Характеризует рентабельность применения препарата, отвечая на вопросы:

Сколько стоит единица эффективности (например, продление жизни на один год) в лечении заболевания при применении препарата?

Сколько потребуется средств для оплаты лечения пациентов данным препаратом?

Инновационный:

Новые технологии, открытые или внедренные при разработке данного препарата,

способствуют как научному прогрессу в целом, так и могут найти свое практическое применение, например, существенно облегчив разработку других препаратов

Этический:

На сколько приемлемо применение рассматриваемого препарата с моральноэтической позиции

Удобство применения:

Насколько удобен препарат в применении, например, каких условий требует введение препарата - стационарных или амбулаторных, требуется ли дополнительное оборудование для введения препарата.
\end{abstract}

При этом в качестве орсранного лекарственного препарата для терапии туберозного склероза рассматривался препарат эверолимус. Опросник для определения весов критериев включал три блока. В первом блоке респондентам предлагалось указать, как по их мнению соотносятся по значимости группы критериев, характеризующие заболевание и лекарственный препарат. Опции ответов были следующими:

- большее значение имеют характеристики заболевания

- большее значение имеют характеристики лечения заболевания

- характеристики заболевания и характеристики лечения заболевания важны в равной степени.

Во втором и в третьем блоках опросника респондентам предлагалось самостоятельно определить вес критериев, характеризующих заболевание и препарат, соответственно. Оценка проводилась посредством присваивания ранга весов каждому критерию. С учетом числа критериев - пяти, как в группе критериев, характеризующих заболевание, так и в группе критериев, характеризующих лечение (препарат), для оценки было доступно пять рангов весов. При этом значение каждого ранга весов было уникальным и не могло повторяться. Наиболее важному критерию соответствовал ранг весов 5, наименее важному - ранг весов 1.

Опросник для оценки туберозного склероза и его лечения по выбранным критериям содержал в себе название критерия и его описание 
и предполагал оценку от 1 до 7 баллов. Один балл (1) отражал слабую позицию описываемого заболевания/препарата по данному критерию, оценка в семь баллов (7) соответствовала сильной характеристике объекта исследования по выбранному критерию.

Таким образом, необходимо отметить, что значения веса критерия и оценки объектов исследования по критериям имели одинаковую шкалу для всех критериев.

Следующим этапом описываемого исследования было проведение анкетирования респондентов с помощью разработанного опросника. В соответствии с методологией были выделены следующие группы респондентов:

Представители пациентов с туберозным склерозом ${ }^{1}$ (Пациентская организация : Межрегиональная Благотворительная общественная организация «Ассоциация больных туберозным склерозом» (зарегистрирована в РФ 1996 году) входит в состав «Общероссийского благотворительного общественного фонда «СОДРУЖЕСТВО»);

Клинические специалисты, осуществляющие лечение туберозного склероза;

Специалисты по оценке технологий здравоохранения и фармакоэкономике;

Лица, принимающие решения в области лекарственного обеспечения (организаторы здравоохранения).

Учитывая доступные организационные ресурсы, опрос в группах представителей пациентов, клинических специалистов и специалистов по оценке технологий здравоохранения был проведен в форме индивидуальных очных интервью. Анкетирование организаторов здравоохранения, представляющих различные субъекты Российской Федерации, проводилось с использованием электронных технологий.

Опрос представителей пациентов и клинических специалистов проводился на базе Научно-исследовательского клинического института педиатрии ГБОУ ВПО «РНИМУ им Н.И.Пирогова, в котором осуществляется наблюдение и лечение пациентов детского возраста с туберозным склерозом. В анкетировании со стороны пациентского сообщества приняло участие 9 представителей (родители детей с туберозным склерозом). В анкетировании приняли участие следующие клинические специалисты (8 человек): два несролога, кардиолог, онко-уролог, четыре невролога. Среди анкетируемых клинических специалистов 6 человек имело ученую степень кандидата медицинских наук, и один доктор медицинских наук.

В проводимом исследовании были опрошены 5 специалистов по оценке технологий здравоохранения, среди которых было 3 кандидата

1 Принимая во внимание многообразие форм туберозного склероза и высокую клиническую значимость терапии эверолимусом пациентов детского возраста, фокус в опросе был направлен именно на эту подгруппу пациентов. фармацевтических наук, доктор фрармацевтических наук и доктор экономических наук.

\section{Результаты проведенного анкетирования}

Результаты опроса показали, что подавляющая доля (более 95\%) респондентов считает в равной мере значимыми как критерии заболевания, так и критерии, характеризующие лечение. В связи с выраженной индиффферентностью респондентов вопрос о соотношении значимости критериев заболевания и лекарственного препарата далее в анализе не рассматривался.

\section{Определение веса отдельных критериев}

Представленные данные в таблице 3 демонстрируют высокую гетерогенность и вариативность полученных ответов. Принимая во внимание, что настоящее исследование является первым в России случаем практического применения МАПР, нами не применялись инструменты, повышающие гомогенность получаемыхданных, такие как коэффициенты согласованности. Также выявленная гетерогенность собранных данных отражает особенности индивидуального интервьюирования, которая являлась основной техникой анкетирования. Наибольшей значимостью среди критериев, характеризующих заболевание, для респондентов групп клинических специалистов, представителей пациентского сообщества и специалистов по ОТЗ и фрармакоэкономике обладал критерий клинической значимости заболевания ( соответственно равны 4,$75 ; 5,00$ и 4,40), в то время как для организаторов здравоохранение наибольшее значение имел экономический критерий $(=4,60)$. В то же время второе место по значимости из факторов, характеризующих заболевания, по мнению респондентов групп клинических специалистов, специалистов по ОТЗ и фармакоэкономике имел экономический критерий, тогда как представители пациентского сообщества поставили его на предпоследнее место по значимости, отдав предпочтение инвестиционному и социальному критериям.

Для всехгрупп респондентов важнейшим фактором, характеризующим лекарственный препарат, оказался клинический фрактор. Представляет интерес распределение весов оставшихся критериев данной группы: на фоне того, что представители пациентских сообществ, специалистов ОТЗ и фрармакоэкономики и организаторов здравоохранения поставили на второе место фрармакоэкономический критерий, клинические специалисты пометили его на последнее место. Полное распределение критериев по значимости представлено в таблице 4.

Результаты оценки туберозного склероза и его лечения по заданным критериям

Анализ полученных оценок для туберозного склероза и препарата эверолимус по заданным критериям позволил выявить достаточно

Таблица 3. Оценки веса критериев критерии в соответствии с мнением различных групп респондентов (баллы)

\begin{tabular}{|c|c|c|c|c|}
\hline Группа респондентов & Клинические специалисты & Пациентское сообщество & Специалисты по 0Т3 & $\begin{array}{c}\text { Организаторы } \\
\text { здравоохранения }\end{array}$ \\
\hline \multicolumn{5}{|l|}{ Критерий } \\
\hline Клинический (заболевание) & 4,75 & 5,00 & 3,80 & 3,80 \\
\hline Экономический & 3,88 & 2,22 & 4,60 & 4,60 \\
\hline Инвестиционный & 2,75 & 3,11 & 2,00 & 2,00 \\
\hline Социальный & 1,88 & 2,78 & 2,40 & 2,40 \\
\hline Эпидемиологический & 1,75 & 1,89 & 2,20 & 2,20 \\
\hline $\begin{array}{c}\text { Клинические } \\
\text { (лекарственный препарат) }\end{array}$ & 4,63 & 4,79 & 4,40 & 4,40 \\
\hline Фармакоэкономический & 2,13 & 3,33 & 4,20 & 4,20 \\
\hline Инновационный & 3,00 & 3,11 & 3,00 & 3,00 \\
\hline Этический & 2,38 & 1,44 & 1,40 & 1,40 \\
\hline Удобство применения & 3,00 & 2,33 & 2,00 & 2,00 \\
\hline
\end{tabular}


Таблица 4. Распределение критериев по значимости в соответствии с мнением различных групп респондентов

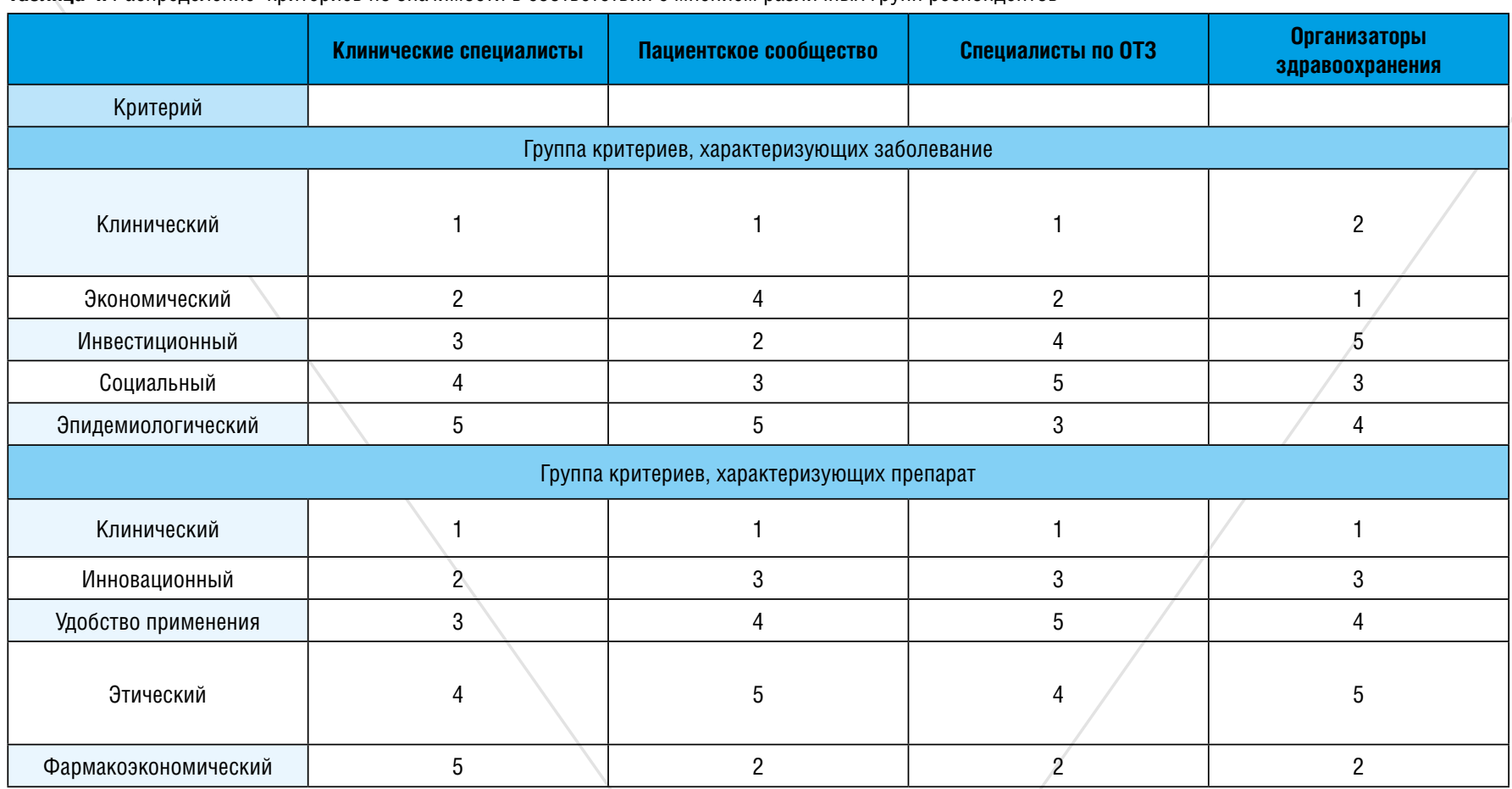

интересные особенности (табл. 5). В частности, клинические специалисты и представители пациентского сообщества чаще использовали высшие оценки - суммарный балл по всем критериям составил для них 56,38 и 56,56 балла соответственно. При этом, представители пациентской организаций несколько выше оценивали само заболевание, туберозный склероз, тогда как клинические специалисты - более чем пациенты оценили лекарственный препарат эверолимус. Специалисты, занимающиеся оценкой технологий здравоохранения, как и организаторы здравоохранения, системно давали более сдержанные оценки по всем критериям, что отразилось в сумме баллов в размере 49,4 и 52,2 балла соответственно. Также необходимо отметить, что суммарный балл как у специалистов по оценке технологий здравоохранения, так и у организаторов здравоохранения был выше для препарата эверолимус, чем для самого заболевания. Оценка заболевания и препарата по критериям отличается от присвоенных критериям весов, что следует из сопоставления данных, приведенных в таблицах 5 и 6. Отдельно представляется интересным проанализировать диапазон средних значений оценок для различных групп респондентов (определялся как разность между самой высокой и самой низкой средней оценкой в группе респондентов). Так, максимальный она была для группы специалистов по ОТЗ - 3,00; в группе клинических специалистов 2,87 ; в группе организаторов здравоохранения 2,80; в то время как в группе представителей пациентских сообществ она составила 2,33. Этот факт может свидетельствовать о ясном видении приоритетов у специалистов здравоохранения и необходимости дополнительного информирования представителей пациентских сообществ о различных аспектах лекарственного обеспечения редких заболеваний.

Результаты интегральной оценки туберозного склероза и препарата

Таблица 5. Оценки туберозного склероза и его лечения по заданным критериям критерии в соответствии с мнением различных групп респондентов (баллы)

\begin{tabular}{|c|c|c|c|c|}
\hline Группа респондентов & Клинические специалисты & Пациентское сообщество & Специалисты по ОТЗ & $\begin{array}{c}\text { Организаторы } \\
\text { здравоохранения }\end{array}$ \\
\hline \multicolumn{5}{|l|}{ Критерий } \\
\hline Клинический (заболевание) & 6,25 & 7,00 & 6,00 & 6,00 \\
\hline Экономический & 4,13 & 4,67 & 5,40 & 4,80 \\
\hline Инвестиционный & 5,00 & 5,56 & 3,60 & 4,20 \\
\hline Социальный & 6,13 & 5,22 & 4,60 & 5,20 \\
\hline Эпидемиологический & 5,50 & 4,78 & 3,60 & 3,60 \\
\hline $\begin{array}{c}\text { Клинические } \\
\text { (лекарственный препарат) }\end{array}$ & 7,00 & 6,56 & 6,60 & 6,40 \\
\hline Фармакоэкономический & 5,00 & 6,00 & 5,20 & 5,20 \\
\hline Инновационный & 5,88 & 6,00 & 5,40 & 4,60 \\
\hline Этический & 6,00 & 4,67 & 4,60 & 6,20 \\
\hline Удобство применения & 5,50 & 6,11 & 4,40 & 6,00 \\
\hline итого & 56,38 & 56,56 & 49,40 & 52,20 \\
\hline
\end{tabular}


Таблица 6. Распределение критериев в соответствии с полученными оценками для туберозного склероза и препарата эверолимус по мнению различных групп респондентов

\begin{tabular}{|c|c|c|c|c|}
\hline & Клинические специалисты & Пациентское сообщество & Специалисты по ОТЗ & $\begin{array}{c}\text { Организаторы } \\
\text { здравоохранения }\end{array}$ \\
\hline Критерий & & & & \\
\hline \multicolumn{5}{|c|}{ Группа критериев, характеризующих заболевание } \\
\hline Клинический & 1 & 1 & 1 & 1 \\
\hline Экономический & 5 & 5 & 2 & 3 \\
\hline Инвестиционный & 4 & 2 & 4,5 & 4 \\
\hline Социальный & 2 & 3 & 3 & 2 \\
\hline Эпидемиологический & 3 & 4 & 4,5 & 5 \\
\hline \multicolumn{5}{|c|}{ Группа критериев, характеризующих препарат } \\
\hline Клинический & 1 & 1 & 1 & 1 \\
\hline Инновационный & 3 & 3,5 & 2 & 5 \\
\hline Удобство применения & 4 & 2 & 5 & 3 \\
\hline Этический & 2 & 5 & 4 & 2 \\
\hline Фармакоэкономический & 5 & 3,5 & 3 & 4 \\
\hline
\end{tabular}

\section{эверолимус}

На заключительном этапе была получена интегральная оценка МАПР для заболевания туберозный склероз и лекарственного препарата эверолимус по заданным критериям посредством умножения среднего значения веса критерия на соответствующую среднюю оценку (рис. 4-5).

Как следует из данных, представленных на рисунке 6, выявленная тенденция к более высоким оценкам представителей пациентского сообщества и клинических специалистов прослеживается и в суммарной интегральной оценке (компенсирующий подход), которая составила для указанных групп респондентов 172,40 и 172,44 балла, соответственно. Тогда как общий балл интегральной оценки специалистами по оценке технологий здравоохранения составил 154,96 баллов, а организаторами здравоохранения - 156,44 балла. Интегральная оценка по отдельным критериям показывает (некомпенсирующий подход), что для всех групп приоритетными критериями, как для заболевания, так и для препарата являются клинические особенности, получившие наивысшие баллы.

Удобной наглядной демонстрацией направления векторов интегральных оценок в разрезе отдельных критериев и групп респондентов является диаграмма «роза ветров» (рис. 7). Каждая из групп респондентов имеет свои отличительные пики в интегральной оценке МАПР по критериям: группа клинических специалистов дала высокие оценки этическому и экономическому критериям туберозного склероза. Клинические специалисты, как отмечалось выше, недооценили фармакоэкономический критерий для лекарственного препарата эверолимус. Группа представителей пациентских организаций имела усредненный профиль оценок без выраженных пиков с небольшим завышением по величине интегральной оценки МАПР по инвестиционному критерию для эверолимуса. Интегральная оценка МАПР специалистами ОТЗ имела характерные особенности по экономическому критерию туберозного склероза (относительно высокое значение), а также по этическому и социальному критериям (относительно низкие значения).

Полученные интегральные оценки свидетельствуют как о понимании жизнеугрожающего статуса туберозного склероза всеми принявшими участие в анализе респондентами, так и высокой эффрективности лекарственного препарата эверолимус в лечении данного заболевания. Схожий уровень оценки по критерию инновационности лекарственного препарата эверолимус отражает согласие всех групп респондентов с инновационным потенциалом данного препарата. В совокупности с достаточно высокой оценкой лицами, принимающими решения, социального критерия, а клиническими специалистами этического критерия, результаты демонстрируют существующую острую потребность в обеспечении пациентов с туберозным склерозом эверолимусом.

\section{Заключение}

Впервые в России был проведен мультикритериальный анализ принятия решений для терапии туберозного склероза препаратом эверолимус. Идентифицированы и описаны позиции заинтересованных сторон в процессе лекарственного обеспечения при туберозном склерозе - как в вопросе об оценке самого заболевания, так и лекарственного препарата.

В результате анализа было установлено, что в отличие от специалистов по оценке технологий здравоохранения и организаторов здравоохранения среди представителей пациентского сообщества и клинических специалистов отмечена тенденция к более высоким оценкам рассмотренных критериев.

Интегральная оценка по отдельным критериям показывает, что для всех групп приоритетными критериями являются клинические особенности, получившие наивысшие баллы. Как представители пациентского сообщества, так и организаторы здравоохранения придали достаточно важное значение критерию социальной значимости туберозного склероза, поставив его на третье место по значимости среди критериев, характеризующих заболевание. Представленные оценки МАПР также свидетельствуют о высокой востребованности препарата эверолимус в лечении пациентов с туберозным склерозом.

Фармакоэкономический критерий у клинических специалистов представляется недооцененным. Также все анкетируемые группь достаточно высоко оценили эверолимус по инновационному критерию.

Выявленные различия в оценках, включенных в анализ критериев, подчеркивают важность учета мнения всех заинтересованных сторонь при принятии решений в области лекарственного обеспечения, особенно больных редкими заболеваниями.

на основе полученных в результате анализа данных можно сделать вывод о понимании важности социального значения рассматриваемого заболевания лицами, ответственными за принятие решений в области лекарственного обеспечения пациентов с редкими заболеваниями.

Таким образом, согласованное мнение всех опрошенных групп респондентов о высокой клинической значимости препарата эверолимус в терапии туберозного склероза отражает выраженную потребность в обеспечении этих пациентов данным препаратом и создает предпосылки 
Интегральная оценка рассматриваемого заболевания и его терапии

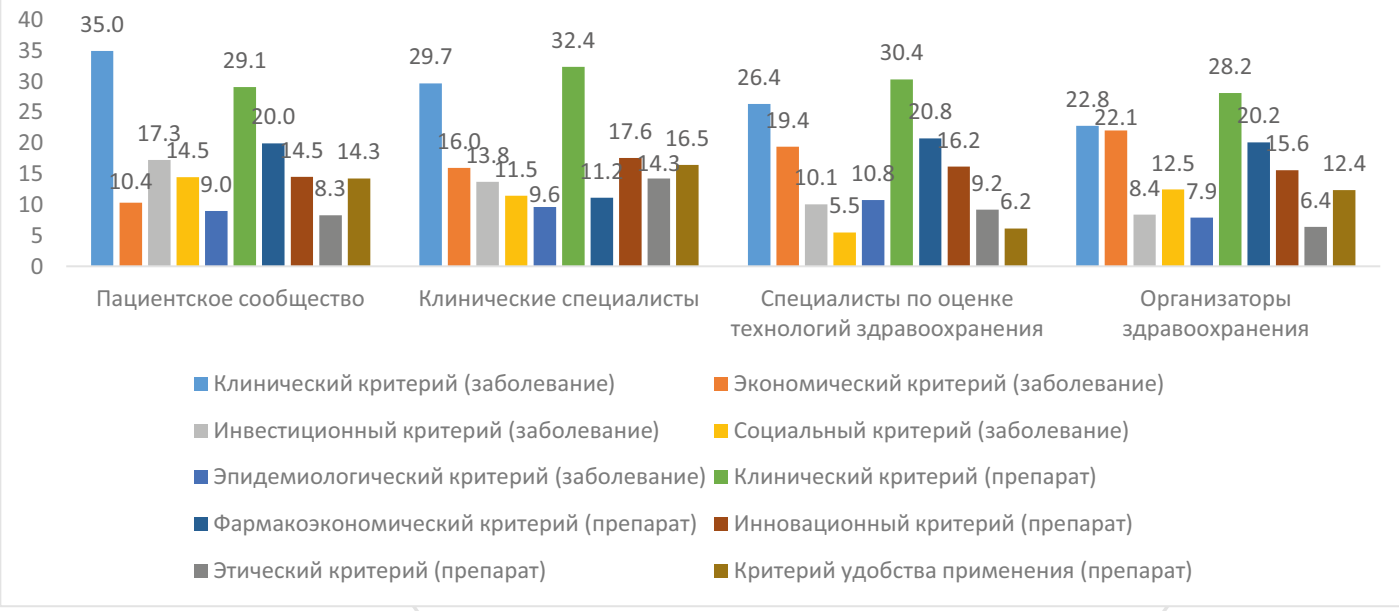

Рисунок 4. Гистограмма интегральной оценки туберозного склероза и лекарственного препарата эверолимус

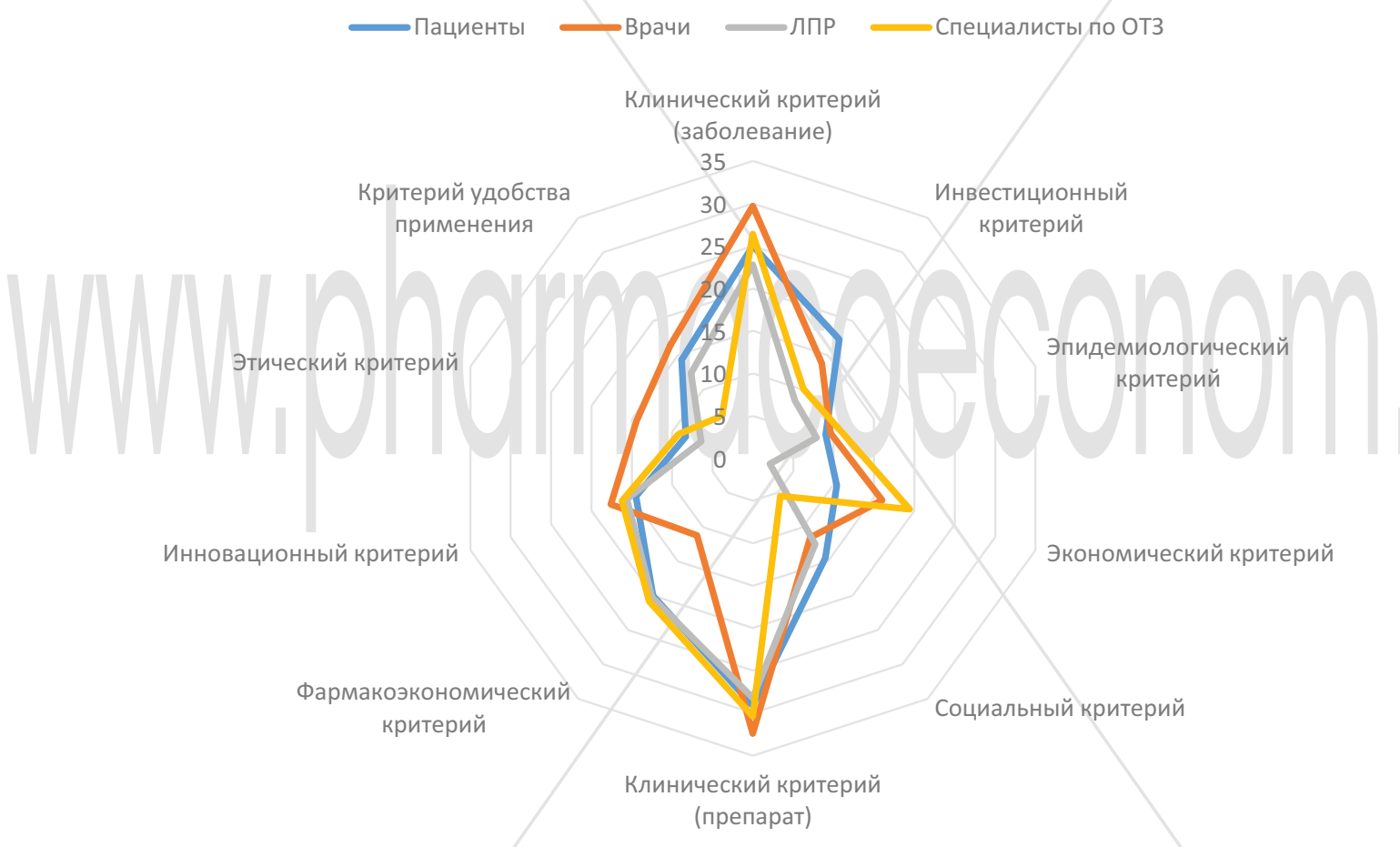

Рисунок 5. Интегральная оценка туберозного склероза и лекарственного препарата эверолимус в форме диаграммы розы ветров

для более гибкого подхода к принятию решений по данной проблеме.

Результаты проведенного исследования могут рассматриваться как дополнительный аргумент при обосновании включения препаратов в перечни лекарственных препаратов, в частности, при обсуждении вопроса лекарственной помощи пациентам с туберозным склерозом.

\section{Список литературы:}

1. Хабриев Р.У., Ягудина Р.И., Правдюк Н.Г. Оценка технологий здравоохранения. - М.: 000 «Медицинское инсоормационное агентство». 2013. $-416 \mathrm{c}$.

2. Ягудина Р.И., Королева Н.И., Зеленова Е.Г. / Редкие заболевания. Орфанные лекарства.// Современная организация лекарственного обеспечения. - 2012. - №1. - С. 6-21.

3. Ягудина Р.И., Зеленова Е.Г., Королева Н.И. / Орфанные заболевания.// Современная организация лекарственного обеспечения. - 2011/2012. - №4/№1. - С. 7-15.

4. Федеральный закон N323-Ф3 от 21.11.2011 «0б основах охраны здоровья граждан в Российской Федерации»

5. Trudy Sullivan. Using MCDA (Multi-Criteria Decision Analysis) to prioritise publicly-funded health care. University of Otago, Dunedin, New Zealand. 2012.

6. Dr. Ling Xu, Dr. Jian-Bo Yang. Introduction to Multi-Criteria Decision Making and the Evidential Reasoning Approach. Manchester School of Management University of Manchester Institute of Science and Technology. 2001.

7. Multi-Criteria Decision Analysis to Value Orphan Medicines. Jon Sussex, Pierrick Rollet, Martina Garau, Claude Schmitt, Alastair Kent, Adam Hutchings. OHE Research Paper. 2013.

8. Combining multicriteria decision analysis, ethics and health technology assessment: applying the EVIDEM decisionmaking framework to growth hormone for Turner syndrome patients. Mireille M Goetghebeur, Monika Wagner, Hanane Khoury, Donna Rindress, Jean-Pierre Grégoire, Cheri Deal. Cost Effectiveness and Resource Allocation 2010, 8:4

9. Nancy j Devlin, Jon Sussex. Incorporating Multiple criteria in HTA. 2011 
10. Tony et al.: Bridging health technology assessment (HTA) with multicriteria decision analyses (MCDA): field testing of the EVIDEM framework for coverage decisions by a public payer in Canada. BMC Health Services Research 2011 11:329.

11. Rob Baltussen, Louis Niessen. Priority setting of health interventions: the need for multi-criteria decision analysis. Cost Effectiveness and Resource Allocation 2006, 4:14

12. Multi-criteria analysis: a manual. Department for Communities and Local Government: London. 2009

13. Multi-criteria decision analysis to prioritize health interventions: Capitalizing on first experiences. Rob Baltussen, Sitapon Youngkong, Francesco Paolucci, Louis Niessen. Health Policy 96 (2010) 262-264

14. The use of multi-attribute utility theory to determine the overall best-inclass performer in a benchmarking study. Terry R. Collins, Manuel D. Rosetti, Heather L. Nachtmann, James R. Oldham. 2004.

15. Quantin Hayez, Yves De Smet, Jimmy Bonney. D-Sight: A New Decision Support System to Address Multi-Criteria Problems. CoDE-SMG \{ Technical Report Series. ISSN 2030-6296

16. Praveen Thokala. Multiple criteria decision analysis for health technology assessment. 2011

17. Государственный реестр лекарственных средств. [Электронный ресурс] - режим доступа: http://grls.rosminzdrav.ru Дата обращения: 10.2015.

18. М.Ю. Дорофеева, Е.Д. Белоусова, А.М. Пивоварова. Рекомендации по диагностике и лечению туберозного склероза. ЖУРНАЛ НЕВРОЛОГИИ И ПСИХИАТРИИ, 3, 2014, с. 58-74

19. Федеральный закон N61-Ф3 от 12.04 .2010 «0б обращении лекарственных средств» 